\title{
Assessing Student Engagement and Higher Order Skill Proficiency During the First and Second Year of Medical School - A Comparison Between the Legacy and Recently Reformed Active Learning Curricula
}

\author{
Brandon Francis ${ }^{1}$, Mari Hopper, $\mathrm{PhD}^{2}$ \\ ${ }^{1}$ Indiana University School of Medicine, ${ }^{2}$ Indiana University School of Medicine, \\ Department of Cellular and Integrative Physiology
}

Background and Hypothesis: This study set-out to determine if:

1. students enrolled in Indiana University's reformed curricula (RC) demonstrate higher levels of engagement (E) and higher order skill (HOS) proficiency than students prior to reform in the legacy curriculum (LC).

2. students increase $E$ and HOS from first year of medical school (MS1) to second year (MS2).

3. students performing in lowest HOS quartile during MS1 will demonstrate greater gains in HOS by MS2 than students in higher quartiles.

Experimental Design or Project Methods: Determined E using a validated selfreport survey (Ahlfeldt, 2007). Assessed HOS using the standardized Collegiate Learning Assessment (CLA+), professionally developed and validated by the Council for Aid to Education (https://cae.org/flagship-assessments-cla-cwra/cla/). Statistical analysis was preliminary; further analysis to be completed by statistician. Between group comparison of LC and RC via t test assuming unequal variance; paired $t$ test for within group comparison MS1 to MS2 (significance $\mathrm{p}<0.05$ ).

Results: Students in RC increased E significantly from MS1 (39.0 \pm 7.0$)$ to MS2 $(40.8 \pm 5.3)$ and demonstrate significantly higher $\mathrm{E}$ than LC MS2 students (36.3 \pm 5.3$)$. There were no differences in HOS proficiency when comparing RC to LC, or MS1 to MS2. Students in RC in the lowest quartile for HOS during MS1 (1688.8 \pm 53.1$)$ significantly increased when re-tested during MS2 (1809.5 \pm 86.8$)$.

Conclusion and Potential Impact: Curricular reform resulted in higher $E$ when compared to LC. Despite increased $\mathrm{E}$, there were no related changes in HOS. Results from quartile analysis agreed with previous reports that active learning preferentially benefits lower performing students (Koles, 2010). 